\title{
Association of genetic variants with myocardial infarction in individuals with or without hypertension or diabetes mellitus
}

\author{
TETSURO YOSHIDA ${ }^{1}$, KIMIHIKO KATO ${ }^{2}$, KIYOSHI YOKOI ${ }^{2}$, MITSUTOSHI OGURI $^{3}$, \\ SACHIRO WATANABE ${ }^{4}$, NORIFUMI METOKI ${ }^{5}$, HIDEMI YOSHIDA $^{6}$, KEI SATOH $^{6}$, \\ YUKITOSHI AOYAGI ${ }^{7}$, YOSHINORI NOZAWA $^{8}$ and YOSHIJI YAMADA ${ }^{9}$ \\ ${ }^{1}$ Department of Cardiovascular Medicine, Inabe General Hospital, Inabe; ${ }^{2}$ Department of Cardiovascular Medicine, \\ Gifu Prefectural Tajimi Hospital, Tajimi; ${ }^{3}$ Department of Cardiology, Japanese Red Cross \\ Nagoya First Hospital, Nagoya; ${ }^{4}$ Department of Cardiology, Gifu Prefectural General Medical Center, Gifu; \\ ${ }^{5}$ Department of Internal Medicine, Hirosaki Stroke Center, Hirosaki; ${ }^{6}$ Department of Vascular Biology, \\ Institute of Brain Science, Hirosaki University Graduate School of Medicine, Hirosaki; \\ ${ }^{7}$ Department of Genomics for Longevity and Health, Tokyo Metropolitan Institute of Gerontology, Tokyo; \\ ${ }^{8}$ Gifu International Institute of Biotechnology and Tokai Gakuin University, Kakamigahara; \\ ${ }^{9}$ Department of Human Functional Genomics, Life Science Research Center, Mie University, Tsu, Japan
}

Received June 5, 2009; Accepted July 30, 2009

DOI: 10.3892/ijmm_00000282

\begin{abstract}
Hypertension and diabetes mellitus are important risk factors for myocardial infarction (MI). The purpose of the present study was to identify genetic variants that confer susceptibility to MI in individuals with or without hypertension or diabetes mellitus, thereby contributing to the personalized prevention of MI in such individuals. The study population comprised 5,835 unrelated Japanese individuals, including 1,339 subjects with MI and 4,496 controls. The 150 polymorphisms were selected by genome-wide association studies of MI and ischemic stroke with the use of the GeneChip Human Mapping 500K Array Set. The genotypes for these polymorphisms were determined by a method that combines the polymerase chain reaction and sequence-specific oligonucleotide probes with suspension array technology. The chi-square test, multivariable logistic regression analysis with adjustment for covariates, as well as a stepwise forward selection procedure revealed that three different polymorphisms were significantly $(\mathrm{P}<0.005)$ associated with the prevalence of MI in individuals with or without hypertension or diabetes mellitus: the $\mathrm{C} \rightarrow \mathrm{T}$ polymorphism of CLEC16A (rs9925481) in individuals without hypertension, the $\mathrm{A} \rightarrow \mathrm{G}$ polymorphism
\end{abstract}

Correspondence to: Dr Yoshiji Yamada, FAHA, Department of Human Functional Genomics, Life Science Research Center, Mie University, 1577 Kurima-machiya, Tsu, Mie 514-8507, Japan E-mail: yamada@gene.mie-u.ac.jp

Key words: genetics, polymorphism, myocardial infarction, diabetes mellitus, hypertension of SEMA3F (rs12632110) in individuals without diabetes mellitus and the $\mathrm{A} \rightarrow \mathrm{G}$ polymorphism of ALOX5 (rs7913948) in individuals without hypertension or diabetes mellitus. No polymorphism was significantly associated with MI in individuals with hypertension, in those with diabetes mellitus, or in those with both conditions. Stratification of subjects based on hypertension or diabetes mellitus may thus be important in order to achieve personalized prevention of MI with the use of genetic information.

\section{Introduction}

Myocardial infarction (MI) is a multifactorial disorder that is thought to result from an interaction between genetic background and environmental factors. Disease prevention is an important strategy for reducing the overall burden of MI, and the identification of markers for disease risk is essential both for risk prediction and for potential intervention to reduce the chance of future events.

Although genetic linkage analyses and association studies have implicated several loci and various candidate genes in predisposition to MI (1), the genes that contribute to genetic susceptibility to MI remain to be identified definitively. We previously showed that genetic variants that confer susceptibility to MI differ between men and women $(2,3)$, between individuals with or without conventional risk factors for atherosclerosis (4), or between individuals with different lipid profiles (5). To further examine whether the association of polymorphisms with MI is influenced by the absence or presence of hypertension or diabetes mellitus, we performed an association study for 150 polymorphisms of 144 candidate genes and MI in 5,835 Japanese individuals with or without hypertension or diabetes mellitus. The purpose of the present study was to identify genetic variants that confer susceptibility 
to MI in individuals with or without hypertension or diabetes mellitus independently and thereby to assess the genetic risk of MI in such individuals separately.

\section{Materials and methods}

Study population. The study population comprised 5,835 unrelated Japanese individuals (3,309 men; 2,526 women) who either visited outpatient clinics of or were admitted to one of the participating hospitals (Gifu Prefectural Tajimi Hospital, Gifu Prefectural General Medical Center, Hirosaki University Hospital, Reimeikyo Rehabilitation Hospital, and Hirosaki Stroke Center) between October 2002 and March 2008 because of various symptoms or for an annual health checkup, or who were recruited to a population-based prospective cohort study of aging and age-related diseases in Gunma Prefecture, Japan. The 1,339 subjects with MI (1,048 men; 291 women) all underwent coronary angiography and left ventriculography. The diagnosis of MI was based on typical electrocardiographic changes and on increases both in the serum activities of enzymes such as creatine kinase, aspartate aminotransferase, and lactate dehydrogenase and in the serum concentration of troponin $\mathrm{T}$. The diagnosis was confirmed by the presence of a wall motion abnormality by left ventriculography and identification of the responsible stenosis in any of the major coronary arteries or in the left main trunk by coronary angiography.

The 4,496 control individuals (2,261 men; 2,235 women) were recruited from community-dwelling healthy individuals or the patients who visited outpatient clinics regularly for treatment of various common diseases. They had no history of MI or coronary heart disease (CHD), ischemic or hemorrhagic stroke, peripheral arterial occlusive disease, other atherosclerotic diseases or other thrombotic, embolic, or hemorrhagic disorders. The subjects with MI and the controls either had or did not have conventional risk factors for CHD, including hypertension (systolic blood pressure of $\geq 140 \mathrm{mmHg}$ or diastolic blood pressure of $\geq 90 \mathrm{mmHg}$, or both, or taking antihypertensive medication) and diabetes mellitus [fasting plasma glucose level of $\geq 6.93 \mathrm{mmol} / \mathrm{l}$ or blood glycosylated hemoglobin (hemoglobin A1c) content of $\geq 6.5 \%$, or both, or taking antidiabetes medication]. On the basis of these criteria, the 2,401 or 3,437 subjects were diagnosed with or without hypertension, respectively, the 1,710 or 4,125 subjects were diagnosed with or without diabetes mellitus, respectively, and the 1,296 or 1,987 subjects were diagnosed with hypertension and diabetes mellitus or without these conditions, respectively.

The study protocol complied with the Declaration of Helsinki and was approved by the Committees on the Ethics of Human Research of Mie University Graduate School of Medicine, Hirosaki University Graduate School of Medicine, Gifu International Institute of Biotechnology, and participating hospitals. Written informed consent was obtained from each participant.

Selection of polymorphisms. A total of 150 polymorphisms (data not shown) were selected by genome-wide association studies of MI and ischemic stroke (P-value for allele frequency $\left.<1.0 \times 10^{-7}\right)$ with the use of the GeneChip Human Mapping 500K Array Set (Affymetrix, Santa Clara, CA) (6). We have

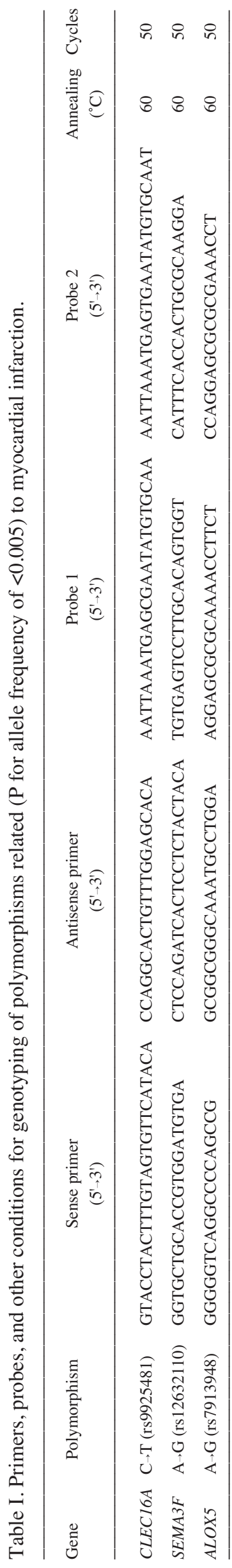


Table II. Characteristics of subjects with myocardial infarction (MI) and controls among individuals with or without hypertension.

\begin{tabular}{|c|c|c|c|c|c|c|}
\hline \multirow[b]{2}{*}{ Characteristic } & \multicolumn{3}{|c|}{ With hypertension } & \multicolumn{3}{|c|}{ Without hypertension } \\
\hline & MI & Controls & $\mathrm{P}$ & MI & Controls & $\mathrm{P}$ \\
\hline No. of subjects & 386 & 2,015 & & 953 & 2,481 & \\
\hline Age (years) & $67.1 \pm 9.6$ & $68.3 \pm 9.8$ & 0.0002 & $64.4 \pm 11.1$ & $65.5 \pm 11.2$ & 0.0083 \\
\hline Sex (male/female, \%) & $75.9 / 24.1$ & $54.7 / 45.3$ & $<0.0001$ & $84.2 / 15.8$ & $44.9 / 55.1$ & $<0.0001$ \\
\hline Body mass index $\left(\mathrm{kg} / \mathrm{m}^{2}\right)$ & $23.9 \pm 3.3$ & $23.7 \pm 3.4$ & 0.0837 & $24.1 \pm 3.1$ & $23.0 \pm 3.2$ & 0.0029 \\
\hline Current or former smoker $(\%)$ & 24.1 & 21.3 & 0.0772 & 27.5 & 23.0 & 0.0577 \\
\hline Systolic blood pressure (mmHg) & $151 \pm 25$ & $150 \pm 24$ & 0.1297 & $121 \pm 14$ & $129 \pm 17$ & $<0.0001$ \\
\hline Diastolic blood pressure $(\mathrm{mmHg})$ & $77 \pm 15$ & $82 \pm 15$ & $<0.0001$ & $68 \pm 11$ & $75 \pm 11$ & $<0.0001$ \\
\hline Hypercholesterolemia (\%) & 59.4 & 36.1 & $<0.0001$ & 25.9 & 27.9 & 0.4129 \\
\hline Serum total cholesterol $(\mathrm{mmol} / \mathrm{l})$ & $5.20 \pm 1.02$ & $5.18 \pm 1.03$ & 0.2468 & $5.18 \pm 1.13$ & $5.17 \pm 0.93$ & 0.4239 \\
\hline Serum triglyceride $(\mathrm{mmol} / \mathrm{l})$ & $1.77 \pm 1.00$ & $1.68 \pm 1.16$ & 0.0001 & $1.68 \pm 1.34$ & $1.48 \pm 0.94$ & 0.0052 \\
\hline Serum HDL-cholesterol (mmol/l) & $1.21 \pm 0.34$ & $1.37 \pm 0.40$ & $<0.0001$ & $1.19 \pm 0.35$ & $1.49 \pm 0.39$ & $<0.0001$ \\
\hline Serum LDL-cholesterol (mmol/l) & $3.19 \pm 0.92$ & $3.04 \pm 0.93$ & $<0.0001$ & $3.15 \pm 0.93$ & $2.99 \pm 0.80$ & 0.0031 \\
\hline Diabetes mellitus (\%) & 51.4 & 32.5 & $<0.0001$ & 40.2 & 12.9 & $<0.0001$ \\
\hline Fasting plasma glucose $(\mathrm{mmol} / \mathrm{l})$ & $7.74 \pm 3.53$ & $6.96 \pm 3.07$ & $<0.0001$ & $7.22 \pm 3.24$ & $6.18 \pm 2.47$ & $<0.0001$ \\
\hline Blood glycosylated hemoglobin (\%) & $6.61 \pm 1.80$ & $5.91 \pm 1.50$ & $<0.0001$ & $6.66 \pm 1.93$ & $5.48 \pm 1.22$ & $<0.0001$ \\
\hline Serum creatinine $(\mu \mathrm{mol} / \mathrm{l})$ & $95.7 \pm 110.3$ & $78.1 \pm 76.2$ & $<0.0001$ & $78.2 \pm 26.5$ & $66.1 \pm 19.3$ & $<0.0001$ \\
\hline
\end{tabular}

Table III. Genotype distribution of SNPs significantly (P-value for allele frequency $<0.005$ ) associated with myocardial infarction (MI) among individuals with or without hypertension as determined by the chi-square test.

\begin{tabular}{|c|c|c|c|c|c|}
\hline Gene symbol & SNP & $\mathrm{dbSNP}$ & MI & Controls & $\mathrm{P}$ (allele frequency) \\
\hline \multicolumn{6}{|c|}{ With hypertension } \\
\hline \multirow[t]{4}{*}{ DNAJC6 } & $\mathrm{C} \rightarrow \mathrm{T}$ & rs2477786 & & & 0.0028 \\
\hline & $\mathrm{CC}$ & & $599(63.0)$ & $1,669(68.0)$ & \\
\hline & $\mathrm{CT}$ & & 303 (31.9) & $697(28.4)$ & \\
\hline & $\mathrm{TT}$ & & $48 \quad(5.1)$ & $90 \quad(3.6)$ & \\
\hline \multicolumn{6}{|c|}{ Without hypertension } \\
\hline \multirow[t]{4}{*}{ CLEC16A } & $\mathrm{C} \rightarrow \mathrm{T}$ & rs9925481 & & & 0.0004 \\
\hline & $\mathrm{CC}$ & & $317(83.0)$ & $1466(73.8)$ & \\
\hline & $\mathrm{CT}$ & & $59(15.4)$ & $482(24.3)$ & \\
\hline & TT & & $6(1.6)$ & 37 (1.9) & \\
\hline \multirow[t]{4}{*}{ ALOX5 } & $\mathrm{A} \rightarrow \mathrm{G}$ & rs7913948 & & & 0.0047 \\
\hline & $\mathrm{AA}$ & & 7 (1.9) & $64(3.2)$ & \\
\hline & $\mathrm{AG}$ & & $89(23.5)$ & $587(29.3)$ & \\
\hline & GG & & $282(74.6)$ & $1352(67.5)$ & \\
\hline
\end{tabular}

Numbers in parentheses are percentages. SNP, single nucleotide polymorphism.

not examined the relation of these polymorphisms to $\mathrm{MI}$ in our previous studies $(2,3,7)$.

Genotyping of polymorphisms. Venous blood $(7 \mathrm{ml})$ was collected into tubes containing $50 \mathrm{mmol} / \mathrm{l}$ ethylenediaminetetraacetic acid (disodium salt), and genomic DNA was isolated with a kit (Genomix; Talent, Trieste, Italy). Genotypes of the 150 polymorphisms were determined at G\&G Science (Fukushima, Japan) by a method that combines the polymerase chain reaction and sequence-specific oligonucleotide probes with suspension array technology (Luminex, Austin, TX). Primers, probes, and other conditions for genotyping of polymorphisms significantly associated with MI are shown in Table I. Detailed genotyping methodology was described previously (8).

Statistical analysis. Quantitative data were compared between subjects with MI and controls by the unpaired Student's t-test. Categorical data were compared by the chisquare test. Allele frequencies were estimated by the gene 
counting method, and the chi-square test was used to identify departures from the Hardy-Weinberg equilibrium. In the initial screen, the allele frequencies of each polymorphism were compared between subjects with MI and controls by the chi-square test. Polymorphisms with a P-value for allele frequency of $<0.005$ were further examined by multivariable logistic regression analysis with adjustment for covariates. Multivariable logistic regression analysis was thus performed with $\mathrm{MI}$ as a dependent variable and independent variables including age, sex $(0$, woman; 1 , man), body mass index (BMI), smoking status ( 0 , nonsmoker; 1 , smoker), serum concentration of creatinine, history of hypertension, diabetes mellitus, or hypercholesterolemia ( 0 , no history; 1 , positive history), and genotype of each polymorphism; and the P-value, odds ratio, and $95 \%$ confidence interval were calculated. Each genotype was assessed according to dominant, recessive, and additive genetic models. Additive models included the additive 1 (heterozygotes versus wild-type homozygotes) and additive 2 (variant homozygotes versus wild-type homozygotes) models, which were analyzed simultaneously with a single statistical model. We also performed a stepwise forward selection procedure to examine the effects of genotypes as well as of other covariates on MI. Each genotype was examined according to a dominant or recessive model on the basis of statistical significance in the multivariable logistic regression analysis. The P-levels for inclusion in and exclusion from the model were 0.25 and 0.1 , respectively. Given the multiple comparisons of genotypes with MI, we adopted the criterion of a P-value of $<0.005$ was considered statistically significant. For other clinical background data, a P-value of $<0.05$ was considered statistically significant. Statistical significance was examined by two-sided tests performed with JMP version 6.0 and JMP Genomics version 3.2 software (SAS Institute, Cary, NC).

\section{Results}

Genetic variants associated with MI in individuals with or without hypertension. The characteristics of the subjects with or without hypertension are shown in Table II. For individuals with hypertension, the frequency of male subjects, serum concentrations of triglycerides, low density lipoprotein (LDL)cholesterol and creatinine, fasting plasma glucose level, blood glycosylated hemoglobin content, and the prevalence of hypercholesterolemia and diabetes mellitus were greater, whereas age, diastolic blood pressure, serum concentration of high density lipoprotein (HDL)-cholesterol were lower, in subjects with MI than in controls. For individuals without hypertension, the frequency of male subjects, BMI, serum concentrations of triglycerides, LDL-cholesterol and creatinine, fasting plasma glucose level, blood glycosylated hemoglobin content, and the prevalence of diabetes mellitus were greater, whereas age, systolic and diastolic blood pressure, serum concentration of HDL-cholesterol were lower, in subjects with MI than in controls.

Comparison of allele frequencies with the chi-square test revealed that one or two polymorphisms were significantly $(\mathrm{P}<0.005)$ associated with $\mathrm{MI}$ in individuals with or without hypertension, respectively (Table III). Multivariable logistic regression analysis with adjustment for age, sex, BMI, smoking

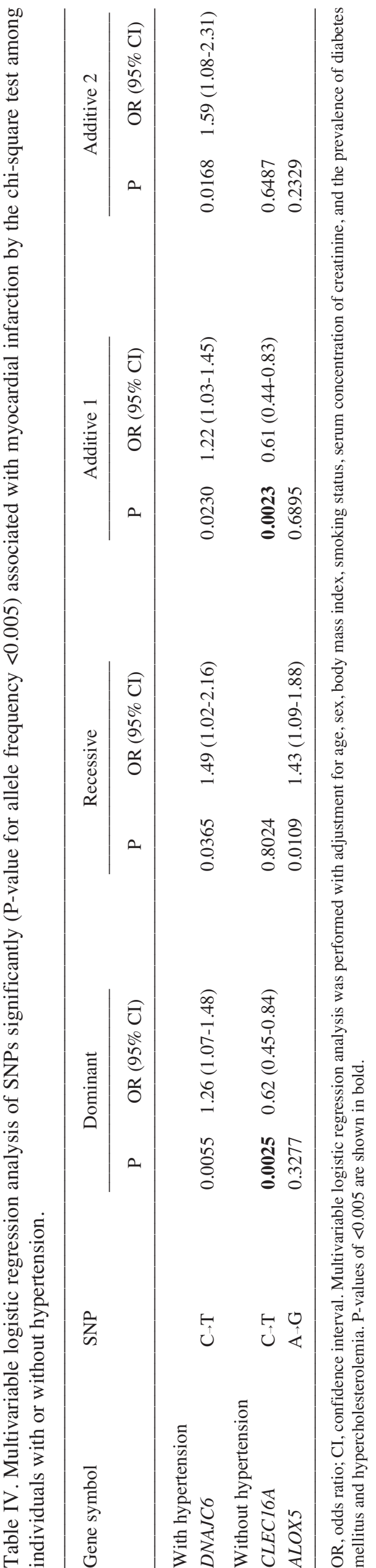


Table V. Characteristics of subjects with myocardial infarction (MI) and controls among individuals with or without diabetes mellitus.

\begin{tabular}{|c|c|c|c|c|c|c|}
\hline \multirow[b]{2}{*}{ Characteristic } & \multicolumn{3}{|c|}{ With diabetes mellitus } & \multicolumn{3}{|c|}{ Without diabetes mellitus } \\
\hline & MI & Controls & $\mathrm{P}$ & MI & Controls & $\mathrm{P}$ \\
\hline No. of subjects & 645 & 1,065 & & 694 & 3,431 & \\
\hline Age (years) & $66.5 \pm 9.6$ & $67.7 \pm 10.1$ & 0.0020 & $66.2 \pm 10.5$ & $66.8 \pm 10.7$ & 0.0434 \\
\hline Sex (male/female, \%) & $79.1 / 20.9$ & $58.2 / 41.8$ & $<0.0001$ & $77.5 / 22.5$ & $47.8 / 52.2$ & $<0.0001$ \\
\hline Body mass index $\left(\mathrm{kg} / \mathrm{m}^{2}\right)$ & $24.1 \pm 3.3$ & $23.7 \pm 3.7$ & 0.0011 & $23.8 \pm 3.2$ & $23.3 \pm 3.2$ & 0.1795 \\
\hline Current or former smoker $(\%)$ & 27.0 & 20.8 & 0.0031 & 23.3 & 22.5 & 0.6168 \\
\hline Hypertension (\%) & 76.0 & 75.7 & 0.8927 & 66.7 & 48.8 & $<0.0001$ \\
\hline Systolic blood pressure (mmHg) & $145 \pm 26$ & $146 \pm 26$ & 0.5051 & $140 \pm 27$ & $140 \pm 23$ & 0.1540 \\
\hline Diastolic blood pressure $(\mathrm{mmHg})$ & $75 \pm 14$ & $80 \pm 15$ & $<0.0001$ & $74 \pm 15$ & $79 \pm 13$ & $<0.0001$ \\
\hline Hypercholesterolemia (\%) & 30.1 & 33.2 & 0.1874 & 28.5 & 28.7 & 0.9490 \\
\hline Serum total cholesterol $(\mathrm{mmol} / \mathrm{l})$ & $5.24 \pm 1.10$ & $5.20 \pm 1.14$ & 0.4488 & $5.16 \pm 1.01$ & $5.17 \pm 0.93$ & 0.9787 \\
\hline Serum triglyceride $(\mathrm{mmol} / \mathrm{l})$ & $1.81 \pm 1.07$ & $1.83 \pm 1.36$ & 0.2188 & $1.68 \pm 1.13$ & $1.52 \pm 0.95$ & $<0.0001$ \\
\hline Serum HDL-cholesterol (mmol/l) & $1.19 \pm 0.34$ & $1.31 \pm 0.38$ & $<0.0001$ & $1.22 \pm 0.03$ & $1.46 \pm 0.40$ & $<0.0001$ \\
\hline Serum LDL-cholesterol (mmol/l) & $3.19 \pm 0.98$ & $3.07 \pm 1.01$ & 0.0123 & $3.17 \pm 0.87$ & $3.00 \pm 0.83$ & $<0.0001$ \\
\hline Fasting plasma glucose (mmol/l) & $9.58 \pm 3.83$ & $9.45 \pm 4.16$ & 0.0603 & $5.53 \pm 0.86$ & $5.70 \pm 1.33$ & 0.4197 \\
\hline Blood glycosylated hemoglobin (\%) & $7.30 \pm 1.90$ & $7.15 \pm 2.02$ & 0.0415 & $5.29 \pm 0.39$ & $5.21 \pm 0.46$ & $<0.0001$ \\
\hline Serum creatinine $(\mu \mathrm{mol} / \mathrm{l})$ & $89.4 \pm 75.7$ & $80.2 \pm 75.6$ & $<0.0001$ & $91.8 \pm 109.1$ & $70.4 \pm 51.6$ & $<0.0001$ \\
\hline
\end{tabular}

Quantitative data are means \pm SD. LDL, low density lipoprotein; HDL, high density lipoprotein.

Table VI. Genotype distributions of SNPs significantly (P-value for allele frequency <0.005) associated with myocardial infarction (MI) among individuals with or without diabetes mellitus as determined by the chi-square test.

\begin{tabular}{|c|c|c|c|c|c|}
\hline Gene symbol & SNP & $\mathrm{dbSNP}$ & MI & Controls & $\mathrm{P}$ (allele frequency) \\
\hline \multicolumn{6}{|c|}{ With diabetes mellitus } \\
\hline \multirow[t]{4}{*}{ PCSK2 } & $\mathrm{C} \rightarrow \mathrm{G}$ & rs6080699 & & & 0.0043 \\
\hline & $\mathrm{CC}$ & & $38(6.0)$ & $97 \quad(9.2)$ & \\
\hline & CG & & $233(36.6)$ & $413(39.2)$ & \\
\hline & GG & & $365(57.4)$ & $544(51.6)$ & \\
\hline \multicolumn{6}{|c|}{ Without diabetes mellitus } \\
\hline \multirow[t]{4}{*}{ SEMA3F } & $A \rightarrow G$ & rs 12632110 & & & 0.0009 \\
\hline & AA & & $185(26.9)$ & $701(20.7)$ & \\
\hline & AG & & $325(47.2)$ & $1692(49.9)$ & \\
\hline & GG & & $178(25.9)$ & $998(29.4)$ & \\
\hline
\end{tabular}

Numbers in parentheses are percentages.

status, serum concentration of creatinine, and the prevalence of diabetes mellitus and hypercholesterolemia revealed that the $\mathrm{C} \rightarrow \mathrm{T}$ polymorphism of CLEC16A (dominant and additive 1 models) was significantly $(\mathrm{P}<0.005)$ associated with $\mathrm{MI}$ in individuals without hypertension (Table IV). No polymorphism was significantly related to MI in individuals with hypertension.

A stepwise forward selection procedure was performed to examine the effects of genotypes for the polymorphism associated with MI by the chi-square test as well as of age, sex, BMI, smoking status, serum concentration of creatinine, and the prevalence of diabetes mellitus and hypercholesterolemia on MI (data not shown). For individuals with hypertension, sex, diabetes mellitus, serum concentration of creatinine, and
DNAJC6 genotype (dominant model), in descending order of statistical significance, were significant $(\mathrm{P}<0.005)$ and independent determinants of MI. For individuals without hypertension, sex, diabetes mellitus, smoking, BMI, and CLEC16A genotype (dominant model), in descending order of statistical significance, were significant and independent determinants of MI.

Genetic variants associated with MI in individuals with or without diabetes mellitus. The characteristics of the subjects with or without diabetes mellitus are shown in Table V. For individuals with diabetes mellitus, the frequency of male subjects, BMI, the percentage of smokers, serum concentrations 
of LDL-cholesterol and creatinine, blood glycosylated hemoglobin content were greater, whereas age, diastolic blood pressure, serum concentration of HDL-cholesterol were lower, in subjects with MI than in controls. For individuals without diabetes mellitus, the frequency of male subject, serum concentrations of triglycerides, LDL-cholesterol, and creatinine, blood glycosylated hemoglobin content, and the prevalence of hypertension were greater, whereas age, diastolic blood pressure, serum concentration of HDL-cholesterol were lower, in subjects with MI than in controls.

Comparison of allele frequencies with the chi-square test revealed that one different polymorphism was significantly $(\mathrm{P}<0.005)$ associated with $\mathrm{MI}$ in individuals with or without diabetes mellitus, respectively (Table VI). Multivariable logistic regression analysis with adjustment for age, sex, BMI, smoking status, serum concentration of creatinine, and the prevalence of hypertension and hypercholesterolemia revealed that the $\mathrm{A} \rightarrow \mathrm{G}$ polymorphism of SEMA3F (dominant model) was significantly $(\mathrm{P}<0.005)$ associated with $\mathrm{MI}$ in individuals without diabetes mellitus (Table VII). No polymorphism was significantly related to MI in individuals with diabetes mellitus.

A stepwise forward selection procedure was performed to examine the effects of genotypes for the polymorphism associated with MI by the chi-square test as well as of age, sex, BMI, smoking status, serum concentration of creatinine, and the prevalence of hypertension and hypercholesterolemia on MI (data not shown). For individuals with diabetes mellitus, sex was a significant $(\mathrm{P}<0.005)$ and independent determinant of MI. For individuals without diabetes mellitus, sex, hypertension, smoking, serum concentration of creatinine, and SEMA3F genotype (dominant model), in descending order of statistical significance, were significant $(\mathrm{P}<0.005)$ and independent determinants of MI.

Genetic variants associated with MI in individuals with hypertension and diabetes mellitus or without these conditions. The characteristics of the subjects with hypertension and diabetes mellitus or without these conditions are shown in Table VIII. For individuals with hypertension and diabetes mellitus, the frequency of male subjects, BMI, the percentage of smokers, fasting plasma glucose level, serum concentration of creatinine were greater, whereas age, diastolic blood pressure, serum concentration of HDL-cholesterol, and the prevalence of hypercholesterolemia were lower, in subjects with MI than in controls. For individuals without hypertension or diabetes mellitus, the frequency of male subjects, serum concentrations of triglycerides and creatinine, and blood glycosylated hemoglobin content were greater, whereas systolic and diastolic blood pressure, and the serum concentration of HDLcholesterol were lower, in subjects with MI than in controls.

Comparison of allele frequencies with the chi-square test revealed that two polymorphisms were significantly $(\mathrm{P}<0.005)$ associated with $\mathrm{MI}$ in individuals without hypertension or diabetes mellitus (Table IX). No polymorphism was significantly related to $\mathrm{MI}$ in individuals with hypertension and diabetes mellitus. Multivariable logistic regression analysis with adjustment for age, sex, BMI, smoking status, serum concentration of creatinine, and the prevalence of hypercholesterolemia revealed that the $\mathrm{A} \rightarrow \mathrm{G}$ polymorphism of ALOX5 (recessive model) was significantly $(\mathrm{P}<0.005)$

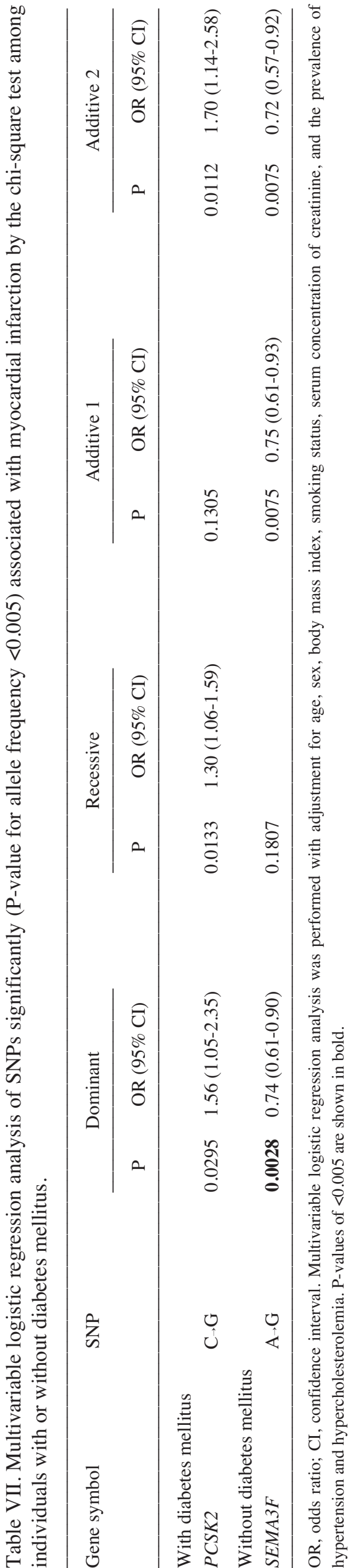


Table VIII. Characteristics of subjects with myocardial infarction (MI) and controls among individuals with hypertension and diabetes mellitus or without these conditions.

\begin{tabular}{|c|c|c|c|c|c|c|}
\hline \multirow[b]{2}{*}{ Characteristic } & \multicolumn{3}{|c|}{ With hypertension and diabetes mellitus } & \multicolumn{3}{|c|}{ Without hypertension or diabetes mellitus } \\
\hline & MI & Controls & $\mathrm{P}$ & MI & Controls & $\mathrm{P}$ \\
\hline No. of subjects & 490 & 806 & & 231 & 1,756 & \\
\hline Age (years) & $67.1 \pm 9.0$ & $68.1 \pm 9.9$ & 0.0236 & $64.4 \pm 11.2$ & $65.3 \pm 11.3$ & 0.0618 \\
\hline Sex (male/female, $\%$ ) & $76.5 / 23.5$ & $59.9 / 40.1$ & $<0.0001$ & $82.3 / 17.7$ & $43.7 / 56.3$ & $<0.0001$ \\
\hline Body mass index $\left(\mathrm{kg} / \mathrm{m}^{2}\right)$ & $24.2 \pm 3.3$ & $23.8 \pm 3.7$ & 0.0105 & $23.3 \pm 3.0$ & $23.0 \pm 3.1$ & 0.1312 \\
\hline Current or former smoker $(\%)$ & 26.5 & 20.1 & 0.0072 & 26.8 & 23.0 & 0.1962 \\
\hline Systolic blood pressure (mmHg) & $152 \pm 24$ & $151 \pm 25$ & 0.2522 & $121 \pm 14$ & $129 \pm 17$ & $<0.0001$ \\
\hline Diastolic blood pressure (mmHg) & $78 \pm 14$ & $81 \pm 15$ & $<0.0001$ & $68 \pm 11$ & $76 \pm 11$ & $<0.0001$ \\
\hline Hypercholesterolemia (\%) & 29.6 & 35.1 & 0.0405 & 22.1 & 28.1 & 0.0546 \\
\hline Serum total cholesterol $(\mathrm{mmol} / \mathrm{l})$ & $5.22 \pm 1.03$ & $5.24 \pm 1.16$ & 0.9918 & $5.10 \pm 1.01$ & $5.19 \pm 0.91$ & 0.0734 \\
\hline Serum triglyceride $(\mathrm{mmol} / \mathrm{l})$ & $1.85 \pm 1.08$ & $1.85 \pm 1.43$ & 0.1125 & $1.62 \pm 1.04$ & $1.44 \pm 0.86$ & 0.0317 \\
\hline Serum HDL-cholesterol (mmol/l) & $1.19 \pm 0.35$ & $1.31 \pm 0.39$ & $<0.0001$ & $1.20 \pm 0.36$ & $1.51 \pm 0.39$ & $<0.0001$ \\
\hline Serum LDL-cholesterol (mmol/l) & $3.17 \pm 0.96$ & $3.12 \pm 1.05$ & 0.2393 & $3.11 \pm 0.86$ & $3.00 \pm 0.79$ & 0.0929 \\
\hline Fasting plasma glucose (mmol/l) & $9.61 \pm 3.83$ & $9.37 \pm 4.05$ & 0.0457 & $5.54 \pm 0.85$ & $5.65 \pm 1.37$ & 0.6376 \\
\hline Blood glycosylated hemoglobin (\%) & $7.21 \pm 1.86$ & $7.05 \pm 1.93$ & 0.0646 & $5.31 \pm 0.40$ & $5.16 \pm 0.46$ & 0.0002 \\
\hline Serum creatinine $(\mu \mathrm{mol} / \mathrm{l})$ & $92.0 \pm 84.8$ & $84.0 \pm 85.8$ & $<0.0001$ & $76.2 \pm 21.9$ & $65.8 \pm 19.0$ & $<0.0001$ \\
\hline
\end{tabular}

Quantitative data are means \pm SD. LDL, low density lipoprotein; HDL, high density lipoprotein.

Table IX. Genotype distributions of SNPs significantly (P-value for allele frequency <0.005) associated with myocardial infarction (MI) among individuals without hypertension or diabetes mellitus as determined by the chi-square test.

\begin{tabular}{|c|c|c|c|c|c|}
\hline Gene symbol & SNP & $\mathrm{dbSNP}$ & MI & Controls & $\mathrm{P}$ (allele frequency) \\
\hline \multirow[t]{4}{*}{ ALOX5 } & $A \rightarrow G$ & rs7913948 & & & 0.0003 \\
\hline & AA & & $4 \quad(1.8)$ & $56(3.2)$ & \\
\hline & $\mathrm{AG}$ & & $43(19.0)$ & $519(29.7)$ & \\
\hline & GG & & $179(79.2)$ & $1174(67.1)$ & \\
\hline \multirow[t]{4}{*}{ CLECI6A } & $\mathrm{C} \rightarrow \mathrm{T}$ & rs9925481 & & & 0.0035 \\
\hline & $\mathrm{CC}$ & & $189(82.9)$ & $1273(73.5)$ & \\
\hline & $\mathrm{CT}$ & & $36(15.8)$ & $426(24.6)$ & \\
\hline & $\mathrm{TT}$ & & $3(1.3)$ & 33 (1.9) & \\
\hline
\end{tabular}

Numbers in parentheses are percentages.

associated with MI in individuals without hypertension or diabetes mellitus (Table X).

A stepwise forward selection procedure was performed to examine the effects of genotypes for the polymorphism associated with MI by the chi-square test as well as of age, sex, BMI, smoking status, serum concentration of creatinine, and the prevalence of hypercholesterolemia on MI (data not shown). For individuals without hypertension or diabetes mellitus, sex, smoking, ALOX5 genotype (recessive model), and CLEC16A genotype (dominant model), in descending order of statistical significance, were significant $(\mathrm{P}<0.005)$ and independent determinants of MI.

Finally, we examined whether the genotype distributions for the polymorphisms associated with MI were in HardyWeinberg equilibrium. The genotype distributions for the $\mathrm{C} \rightarrow \mathrm{T}$ polymorphism of CLEC16A in individuals without hypertension, the $\mathrm{A} \rightarrow \mathrm{G}$ polymorphism of $S E M A 3 F$ in individuals without diabetes mellitus, and the $\mathrm{A} \rightarrow \mathrm{G}$ polymorphism of ALOX5 in individuals without hypertension or diabetes mellitus were all in Hardy-Weinberg equilibrium both in subjects with MI and in controls (data not shown).

\section{Discussion}

We examined the possible relations of 150 polymorphisms of 144 genes to the prevalence of MI in individuals with or without hypertension or diabetes mellitus. Our association study with three steps of analysis (chi-square test, multivariable logistic regression analysis, and stepwise forward selection procedure) revealed that three different polymorphisms were significantly associated with $\mathrm{MI}$ : the $\mathrm{C} \rightarrow \mathrm{T}$ polymorphism of CLEC16A (rs9925481) in individuals 
without hypertension, the $\mathrm{A} \rightarrow \mathrm{G}$ polymorphism of $S E M A 3 F$ (rs12632110) in individuals without diabetes mellitus, and the $\mathrm{A} \rightarrow \mathrm{G}$ polymorphism of $A L O X 5$ (rs7913948) in individuals without hypertension or diabetes mellitus. Our results suggest that genetic variants that confer susceptibility to MI differed among individuals with or without hypertension or diabetes mellitus and that genetic component for the development of MI was more apparent in individuals at low-risk than in those at high-risk.

The C-type lectin domain family 16, member A (CLEC16A) was shown to be highly expressed in B lymphocytes and natural killer and dendritic cells (9). The $\mathrm{G}$ allele of the $\mathrm{G} \rightarrow \mathrm{A}$ polymorphism of CLEC16A (rs2903692) was related to an increased risk for type 1 diabetes mellitus in Japanese subjects $(10)$, as well as in white populations $(11,12)$. Three common non-coding variants of CLEC16A (rs2903692, rs725613, and rs17673553), which were in strong linkage disequilibrium, were significantly associated with type 1 diabetes mellitus in a genome-wide association study (12). These results indicate that CLEC16A is involved in the pathogenesis of type 1 diabetes mellitus. We have now shown that $\mathrm{C} \rightarrow \mathrm{T}$ polymorphism of CLEC16A (rs9925481) was significantly associated with $\mathrm{MI}$ in individuals without hypertension, with the $T$ allele protecting against this condition, although the mechanism responsible for this association remains to be elucidated.

Sema, immunoglobulin and short basic domains, secreted, 3F (SEMA3F) is a secreted member of the semaphorin III family. During the developmental process, SEMA3F plays a pivotal role in axon guidance in the peripheral and central nervous system by interacting with its receptor, neuropilin 2 (NRP2) (13). The SEMA3F-NRP2 signaling pathway guides axonal extension by means of a chemotactic repulsing effect on the axons (14). SEMA3F was isolated from a region of 3 p21.3 involved in homozygous deletions in small cell lung cancer cell lines and was recognized as a candidate tumor suppressor gene, given that p53 inhibits tumor vessel formation and cell growth through the SEMA3F-NRP2 pathway (15). We have now shown that the $\mathrm{A} \rightarrow \mathrm{G}$ polymorphism of $S E M A 3 F$ (rs12632110) was significantly associated with MI in individuals without diabetes mellitus, with the $G$ allele protecting against this condition, although the underlying mechanism remains unclear.

Arachidonate 5-lipoxygenase (ALOX5) is the key enzyme in the oxdative biosynthesis of leukotrienes (16). The ALOX5 pathway has been related to atherosclerosis in mouse models $(17,18)$ and, in a histological study, in humans $(19)$. There is a tandem repeat polymorphism in the promoter of ALOX5. The common allele has five tandem 5'-GGGCGG-3' repeats (20), while the variant allele has fewer or greater number of repeats. In a cohort of 470 middle-aged men and women without overt cardiovascular disease, mean carotid intimamedia thickness (IMT) was increased among carriers of two variant alleles, as compared to subjects carrying at least one common allele (20). Given that increased carotid IMT is a marker of systemic atherosclerosis (21) and is a predictor of future CHD (22), these observations implicate $A L O X 5$ as a candidate gene for CHD. We have now shown that the $A \rightarrow G$ polymorphism of ALOX5 (rs7913948) was significantly associated with the prevalence of MI in individuals without 
hypertension or diabetes mellitus, with the $G$ allele representing a risk factor for this condition. Effects of this polymorphism on the development of atherosclerosis may account for its association with MI.

Our study has limitations: (i) it is possible that one or more of the polymorphisms associated with MI in the present study are in linkage disequilibrium with other polymorphisms in the same gene or in other nearby genes that are actually responsible for the development of this condition, (ii) although we adopted the criterion of $\mathrm{P}<0.005$ for association to compensate for the multiple comparisons of genotypes with MI, it is not possible to exclude completely potential statistical errors such as false positives, (iii) the functional relevance of the identified polymorphisms to gene transcription or to protein structure or function was not determined in the present study.

In conclusion, genetic variants that confer susceptibility to MI differed among individuals with or without hypertension or diabetes mellitus. Stratification of subjects based on hypertension or diabetes mellitus may thus be important in order to achieve personalized prevention of MI with the use of genetic information. Given that our present study may be considered as hypothesis generating, validation of our findings will require their replication with independent subject panels.

\section{Acknowledgements}

This work was supported in part by Grants-in-Aid for Scientific Research from the Ministry of Education, Culture, Sports, Science, and Technology of Japan (nos. 18209023, 18018021, and 19659149 to Y.Y.).

\section{References}

1. Yamada Y, Ichihara S and Nishida T: Molecular genetics of myocardial infarction. Genomic Med 2: 7-22, 2008.

2. Yamada Y, Izawa H, Ichihara S, et al: Prediction of the risk of myocardial infarction from polymorphisms in candidate genes. N Engl J Med 347: 1916-1923, 2002.

3. Yamada Y, Matsuo H, Segawa T, et al: Assessment of genetic risk for myocardial infarction. Thromb Haemost 96: 220-227, 2006.

4. Nishihama K, Yamada Y, Matsuo H, et al: Association of gene polymorphisms with myocardial infarction in individuals with or without conventional coronary risk factors. Int J Mol Med 19: 129-141, 2007.

5. Yoshida T, Yajima K, Hibino T, et al: Association of gene polymorphisms with myocardial infarction in individuals with different lipid profiles. Int J Mol Med 20: 581-590, 2007.
6. Yamada Y, Fuku N, Tanaka M, et al: Identification of CELSR1 as a susceptibility gene for ischemic stroke in Japanese individuals by a genome-wide association study. Atherosclerosis (published online).

7. Yamada Y, Kato K, Oguri M, et al: Genetic risk for myocardial infarction determined by polymorphisms of candidate genes in a Japanese population. J Med Genet 45: 216-221, 2008.

8. Itoh Y, Mizuki N, Shimada T, et al: High-throughput DNA typing of HLA-A, -B, -C, and -DRB1 loci by a PCR-SSOPLuminex method in the Japanese population. Immunogenetics 57: 717-729, 2005.

9. International Multiple Sclerosis Genetics Consortium (IMSGC): The expanding genetic overlap between multiple sclerosis and type I diabetes. Genes Immun 10: 11-14, 2009.

10. Awata T, Kawasaki E, Tanaka S, et al: Association of type 1 diabetes with two Loci on 12q13 and 16p13 and the influence coexisting thyroid autoimmunity in Japanese. J Clin Endocrinol Metab 94: 231-235, 2009.

11. Todd JA, Walker NM, Cooper JD, et al: Robust associations of four new chromosome regions from genome-wide analyses of type 1 diabetes. Nat Genet 39: 857-864, 2007.

12. Hakonarson H, Grant SF, Bradfield JP, et al: A genome-wide association study identifies KIAA0350 as a type 1 diabetes gene. Nature 448: 591-594, 2007.

13. Chen H, Chédotal A, He Z, Goodman CS and Tessier-Lavigne M: Neuropilin-2, a novel member of the neuropilin family, is a high affinity receptor for the semaphorins Sema E and Sema IV but not Sema III. Neuron 19: 547-559, 1997.

14. Chen H, Bagri A, Zupicich JA, et al: Neuropilin-2 regulates the development of selective cranial and sensory nerves and hippocampal mossy fiber projections. Neuron 25: 43-56, 2000.

15. Futamura M, Kamino H, Miyamoto Y, et al: Possible role of semaphorin $3 \mathrm{~F}$, a candidate tumor suppressor gene at $3 \mathrm{p} 21.3$, in p53-regulated tumor angiogenesis suppression. Cancer Res 67: 1451-1460, 2007.

16. Samuelsson B: Leukotrienes: mediators of immediate hypersensitivity reactions and inflammation. Science 220: 568-575, 1983.

17. Mehrabian M, Allayee H, Wong J, et al: Identification of 5-lipoxygenase as a major gene contributing to atherosclerosis susceptibility in mice. Circ Res 91: 120-126, 2002.

18. Aiello RJ, Bourassa PA, Lindsey S, Weng W, Freeman A and Showell HJ: Leukotriene B4 receptor antagonism reduces monocytic foam cells in mice. Arterioscler Thromb Vasc Biol 22: 443-449, 2002.

19. Spanbroek R, Grabner R, Lotzer K, et al: Expanding expression of the 5-lipoxygenase pathway within the arterial wall during human atherogenesis. Proc Natl Acad Sci USA 100: 1238-1243, 2003.

20. Dwyer JH, Allayee H, Dwyer KM, et al: Arachidonate 5-lipoxygenase promoter genotype, dietary arachidonic acid, and atherosclerosis. N Engl J Med 350: 29-37, 2004.

21. Mitchell JR and Schwartz CJ: Relationship between arterial disease in different sites. A study of the aorta and coronary, carotid, and iliac arteries. Br Med J 1: 1293-1301, 1962.

22. Iglesias del Sol A, Bots ML, Grobbee DE, Hofman A and Witteman JC: Carotid intima-media thickness at different sites: relation to incident myocardial infarction; The Rotterdam Study. Eur Heart J 23: 934-940, 2002. 